\title{
Extensive multiplex PCR diagnostics reveal new insights into the epidemiology of viral respiratory infections
}

\author{
S. NICKBAKHSH ${ }^{1}$, F. THORBURN ${ }^{1}$, B. VON WISSMANN ${ }^{2}$, J. MCMENAMIN ${ }^{2}$, \\ R. N. GUNSON ${ }^{3}$ AND P. R. MURCIA ${ }^{1 *}$ \\ ${ }^{1}$ MRC-University of Glasgow Centre for Virus Research, Institute of Infection, Inflammation and Immunity, \\ Glasgow, UK \\ ${ }^{2}$ Health Protection Scotland, NHS National Services Scotland, Glasgow, UK \\ ${ }^{3}$ West of Scotland Specialist Virology Centre, NHS Greater Glasgow and Clyde, Glasgow UK
}

Received 14 July 2015; Final revision 20 November 2015; Accepted 3 February 2016; first published online 2 March 2016

\section{SUMMARY}

Viral respiratory infections continue to pose a major global healthcare burden. At the community level, the co-circulation of respiratory viruses is common and yet studies generally focus on single aetiologies. We conducted the first comprehensive epidemiological analysis to encompass all major respiratory viruses in a single population. Using extensive multiplex PCR diagnostic data generated by the largest NHS board in Scotland, we analysed 44230 patient episodes of respiratory illness that were simultaneously tested for 11 virus groups between 2005 and 2013, spanning the 2009 influenza A pandemic. We measured viral infection prevalence, described coinfections, and identified factors independently associated with viral infection using multivariable logistic regression. Our study provides baseline measures and reveals new insights that will direct future research into the epidemiological consequences of virus co-circulation. In particular, our study shows that (i) human coronavirus infections are more common during influenza seasons and in co-infections than previously recognized, (ii) factors associated with co-infection differ from those associated with viral infection overall, (iii) virus prevalence has increased over time especially in infants aged $<1$ year, and (iv) viral infection risk is greater in the post-2009 pandemic era, likely reflecting a widespread change in the viral population that warrants further investigation.

Key words: Co-infections, pandemic influenza, risk factor analysis, surveillance.

\section{INTRODUCTION}

Acute respiratory infections are the commonest cause of illness in all ages, and a leading cause of mortality in children aged $<5$ years, creating a significant global healthcare burden [1-3]. Various aetiological pathogens (viruses, bacteria and some fungi) are recognized,

\footnotetext{
* Author for correspondence: Dr P. R. Murcia, MRC-University of Glasgow Centre for Virus Research, Institute of Infection, Inflammation and Immunity, Glasgow G61 1QH, UK. (Email: Pablo.Murcia@Glasgow.ac.uk)
}

causing largely indistinguishable symptoms. In most settings, viruses are the most frequently detected agent $[4,5]$. Although most infections are mild, respiratory viruses have the potential to cause severe illness in high-risk groups.

Although influenza is a major research focus [6], the advent of polymerase chain reaction (PCR) technology has led to improved awareness that non-influenza viruses are also important contributors to disease burden, and of the role of viral subtype in clinical severity [7-9]. The use of PCR testing as part of routine diagnostics provides an important resource for monitoring 
respiratory viruses as part of national surveillance [10].

Multiplex PCR methods in particular provide a valuable resource for epidemiological enquiry [11]. All patients requiring microbiological diagnosis are tested for all pathogens included in the panel, ensuring consistency in testing across patients. The collation of multiplex diagnostic data from a large patient population and over an extended time-frame therefore enables robust comparisons of infection trends temporally and across patient subgroups. Furthermore, when testing is implemented over multiple years, sufficient data can be accrued to investigate the clinical relevance of co-infections and their epidemiological patterns [12].

Although the utility of diagnostic data in the epidemiology of respiratory infections has been demonstrated [11, 13-16], studies that cover all major viruses, patient age and illness severity groups, and that span multiple years, are lacking. The largest NHS health board in Scotland, Greater Glasgow and Clyde (NHSGGC), has used multiplex PCR testing as part of their routine diagnostic services since 2005 . This health board serves $\sim 1 \cdot 1$ million people, representing $\sim 1.7 \%$ of the total UK population [17]. The resultant accumulation of data provides a novel opportunity to investigate viral respiratory infections in a more comprehensive fashion than previously possible. These data also provide a unique opportunity to compare the periods before and after the introduction of the novel pandemic influenza virus $[\mathrm{A}(\mathrm{H} 1 \mathrm{~N} 1)$ pdm09] into Scotland (see [18]).

We analysed diagnostic data generated by NHSGGC using multiplex PCR from 2005 to 2013 with the following objectives: (i) to describe testing and virus prevalence trends, (ii) to examine temporal and patient subgroup distributions for each individual virus, and (iii) to compare factors associated with overall viral infection and co-infection using statistical modelling, in order to provide robust and timely estimates of who is most at risk of viral-associated respiratory illness, and when, within a major urban UK population.

\section{METHODS}

\section{Virological data}

In this study we used virological diagnostic data generated by the West of Scotland Specialist Virology Centre (WoSSVC) for NHSGGC during 2005-2013
[19]. During this period, a total of 61427 clinical samples were received from 40962 patients attending primary and secondary healthcare services for respiratory diagnostic purposes (i.e. excluding pathology-origin samples). Most $(98 \%)$ clinical samples were taken from the upper or lower respiratory tract: primarily nasal and/or throat swabs (67\%), gargles $(13 \%)$, nasopharyngeal aspirates $(7 \%)$, sputum $(5 \%)$, bronchoalveolar lavage (3\%) and nasopharyngeal/ endotracheal secretions $(2 \%)$. In a minority of cases ( $n=142$ samples), plasma was additionally taken for follow-up investigation; most (89\%) of these samples related to the 2009 influenza A pandemic period which was excluded from statistical modelling analyses.

Each sample was tested by real-time RT-PCR for 11 groups of respiratory viruses: human rhinovirus (RV); influenza A virus [IAV; a generic assay detecting seasonal $\mathrm{H} 3 \mathrm{~N} 2$ and $\mathrm{H} 1 \mathrm{~N} 1$ subtypes and one specific to $\mathrm{A}(\mathrm{H} 1 \mathrm{~N} 1) \mathrm{pdm} 09$ ], influenza $\mathrm{B}$ virus (IBV), human respiratory syncytial virus (RSV), human coronavirus (CoV; aggregating 229E, NL63, HKU1 and OC43 species), adenovirus (AdV), human metapneumovirus (MPV) and human parainfluenza types 1-4 (PIV1-4). Details of nucleic acid extraction methods and the real-time PCR assays are provided elsewhere [20].

Complete testing coverage across viruses was largely maintained throughout the study period. However, high frequencies of partial testing did arise due to the burden placed on laboratory resources during the major waves of $\mathrm{A}(\mathrm{H} 1 \mathrm{~N} 1)$ pdm09 virus circulation. The laboratory protocols were consistent throughout the study period, with the exception of the RV assay which was modified during 2009 to detect a wider array of RV and enteroviruses (including D68), and the CoV-HKU1 assay which was discontinued in 2012.

\section{Data preparation and descriptive analysis}

For each of the 61427 clinical samples, positive/negative PCR test results were recorded by the laboratory for each virus group. Information was also provided on the sampling date, patient's age at sampling, gender, and the origin of the sample [whether the patient had attended a General Practice (GP), hospital outpatient or non-critical-care inpatient services, or was admitted to a critical care ward]. In the case of inconclusive/absent test results or other patient information, the corresponding data were coded as missing. All patient identifiers were anonymized. 
Of the 40962 patients, 8394 had multiple samples submitted for virological testing during the study period (range 1-37 samples, median 1, S.D. $=1 \cdot 22$ ). For $70 \%$ of these patients, the samples were received within a 30-day window. We aggregated the PCR test results to within this time-frame generating single 'episodes' of respiratory illness, using the collection date of the first sample when assigning temporal information. Episodes were classified as positive for a given virus if at least one sample tested positive. Following data exclusions, 44230 patient episodes, representing 36157 individual patients, were retained for analysis of temporal distributions. We conducted descriptive statistical analyses of viral infection prevalence in the patient population providing time- and agestratified estimates.

By the end of April 2009, Scotland was afflicted by the influenza pandemic [20]. Figure $1 a$ highlights the resultant upsurge in testing frequencies during the summer and autumn waves of 2009, and during a third wave of $\mathrm{A}(\mathrm{H} 1 \mathrm{~N} 1) \mathrm{pdm} 09$ virus circulation in the winter of 2010/2011. During these periods, testing was primarily directed towards IAV and only subsets of IAV-negative patients were tested for other viruses. Due to this disruption in regular testing procedures, we focused our description of viral infection distributions across patient subgroups on the 26974 patient episodes tested outside this period, and refer readers to a previous report for details of viruses detected during the 2009 pandemic [20].

\section{Co-infection analyses}

For each virus group, we compared the frequency of mono-infection episodes (one virus group detected) and co-infection episodes (more than one virus group detected). To correctly classify episodes into these subgroups, we excluded all partially tested patients. In more detailed analyses, we counted the frequency of each possible virus pair and quantified the statistical correlation between mono-infection and co-infection frequencies across viruses.

\section{Statistical associations}

We investigated statistical associations between time period, season, patient age, gender, and GP/general hospital/critical care origin (a proxy for illness severity), and two outcomes: (i) virus-positive vs. virusnegative episodes, and (ii) co-infection vs. monoinfection episodes. With respect to time, we split sampling dates into two major periods either side of the influenza pandemic and periods of high partial testing: pre-pandemic [prior to May 2009 when the A(H1N1)pdm09 virus was established in Scotland] and post-pandemic [following subsidence of the third major wave of the $\mathrm{A}(\mathrm{H} 1 \mathrm{~N} 1)$ pdm09 virus in January 2011].

Associations with each factor were first assessed by crude unadjusted odds ratios, and then adjusted for confounding using multivariable logistic regression models that included all factors to assess their independence. Statistical interactions were examined using Mantel-Haenszel stratification methods (based on $P<0 \cdot 05$, results not shown). The potential interactions were added to the main effects models and their significance assessed based on an interaction parameter $P<0 \cdot 05$. Model fit was assessed by le Cessie van Houwelingen global goodness-of-fit tests [21]. All statistical analyses were carried out in R v. $3 \cdot 1 \cdot 1$ [22].

To correctly classify patients into outcome groups, all partially tested patients were excluded. Of the 36157 fully tested patients, $90 \%$ sought healthcare facilities once during the study period thereby contributing a single episode. However, 4218 patients had attended healthcare facilities more than once, providing information for multiple episodes (range 2-26 episodes, median 2, s.D. $=2 \cdot 04)$. We retained the first observed episode per patient in the statistical analyses to ensure the patient-level interpretation of statistical associations was not influenced by the nonindependence of data relating to the same individual. See Supplementary Figure S1 for full details of data preparation.

\section{RESULTS}

\section{Episodes of illness and viral infection frequencies}

We analysed 44230 episodes of respiratory illness tested by WoSSVC during 2005 to 2013. Full details of patient distributions across subgroups and per study year are provided in Supplementary Table S1. Patients' median age was 27 years (range $0-98$ years, S.D. $=25.5$ years) and $49 \%$ were male. Excluding the three major waves of influenza A(H1N1)pdm09 virus circulation, episode frequencies increased year-by-year from 2472 cases tested in 2005 to 6149 cases tested in 2013. However, the age patterns were not consistent over this period; the percentage of adult episodes was greater in 2013 than in 2005 (e.g. $21 \%$ vs. $8 \%$ in patients aged $\geqslant 65$ years), while the 
(a)

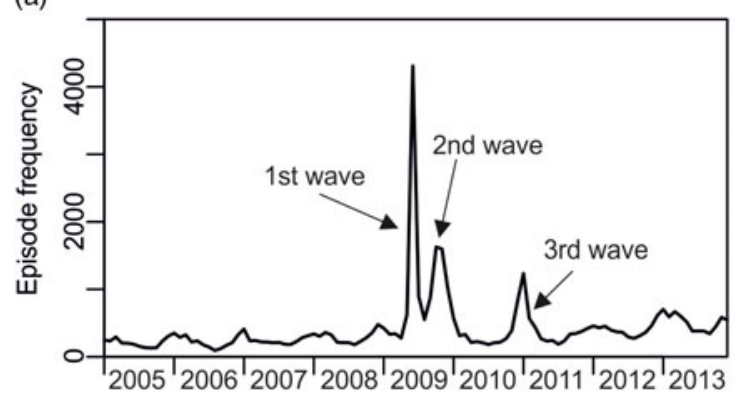

(c)

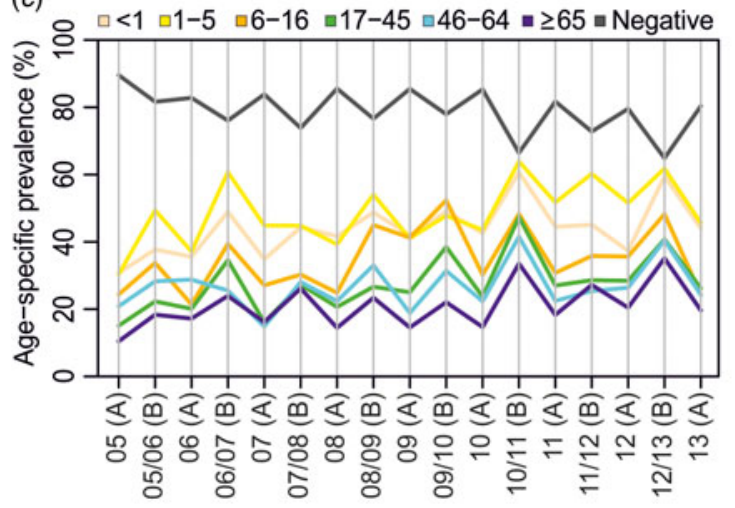

(b)

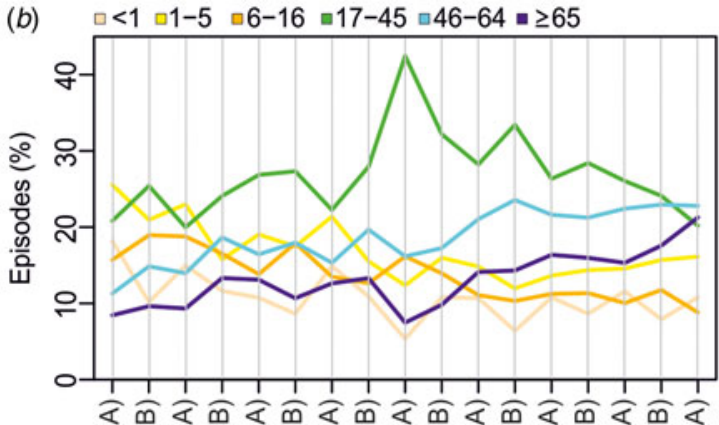

ㄴㅇㅇ

(d)

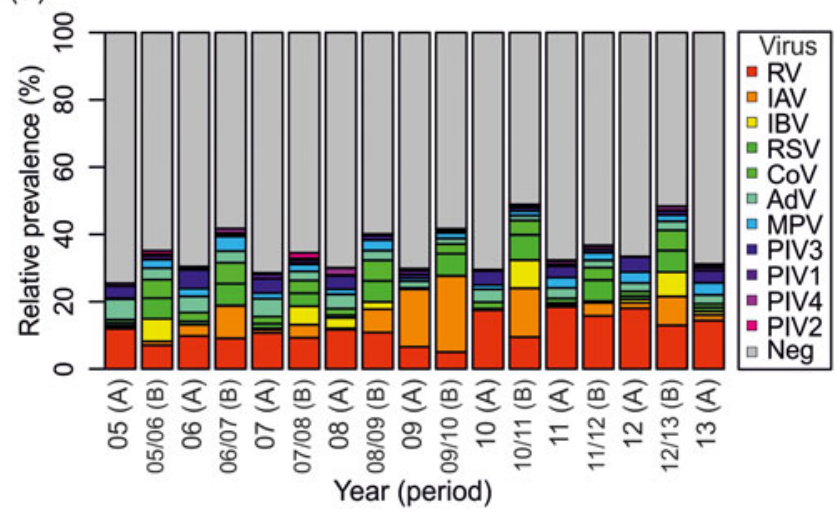

Fig. 1. Trends in episodes of respiratory illness and viral infection prevalence in patients seeking healthcare services within NHS Greater Glasgow and Clyde during 2005-2013. (a) Episodes of respiratory illness tested in each month highlighting the three major waves of $\mathrm{A}(\mathrm{H} 1 \mathrm{~N} 1)$ pdm09 virus circulation. (b) Distribution of episodes across age groups in each 6-month period. (c) Age-specific prevalence of confirned viral infection and virus-negative illness detected in each 6-month period. (d) Relative prevalence of each viral infection and virus-negative illness (Neg) in each 6-month period; A, typical non-influenza period (April-September); B, typical influenza period (October-March). Note that January-March 2005 and October-December 2013 were excluded from panel $(d)$.

percentage of child episodes was fewer in 2013 than 2005 (e.g. $16 \%$ vs. $26 \%$ in patients aged $1-5$ years) (Fig. 1b).

At least one virus was detected in 35\% (15 302/44 230) of tested patients; these patients had a median age of 17 years (range $0-96$ years, s.D. $=25$ years) and $49 \%$ were male. The prevalence of confirmed viral infection in the patient population was greater in the 2013 influenza season than in 2005 in all age groups (Fig. 1c); the absolute difference in prevalences was $22 \%$ (infants aged $<1$ year), $12 \%$ (1-5 years), 14\% (6-16 years), 18\% (17-45 years), 12\% (46-64 years) and $17 \%$ ( $\geqslant 65$ years). Overall virus-specific prevalences in the patient population were ranked as follows: RV $(14 \%, n=4847)$; IAV $(9 \cdot 7 \%, n=4244)$; RSV $(4 \cdot 9 \%, n=1786) ; \operatorname{CoV}(4 \cdot 1 \%, n=1339) ; \mathrm{AdV}$ $(3.6 \%, n=1221)$; IBV $(3 \%, n=1019)$; MPV $(2 \cdot 6 \%$, $n=345)$; PIV-3 (2.2\%, $n=757)$; PIV-4 $(0 \cdot 86 \%, n=286)$; PIV-1 $(0 \cdot 84 \%, n=295)$ and PIV-2 $(0 \cdot 35 \%, n=122)$.
Age distributions for each viral infection group are presented in Supplementary Table S2. The most common infection in each 6-month period (excluding 2009) was $\mathrm{RV}$, constituting a low of $19 \%$ of infections during the typical influenza period of 2005/2006, to a high of $59 \%$ during the typical non-influenza period of 2010 (Fig. 1d).

For most virus groups, detections were most frequent in the 1-5 years group (with the exception of IAV, IBV and CoV), males, and hospital attendees not admitted to a critical care ward (Fig. 2). Seasonally, virus detections were most common in December (45\% in GP attendees, $43 \%$ in hospital attendees) and least common in August (11\% in GP attendees, 22\% in hospital attendees) (Fig. 3a,c). The most commonly detected viral infection in each month was $\mathrm{RV}$, peaking in September in both GP and hospital attendees (Fig. 3b,d). Influenza A and B were the most common detections in December-March in GP 

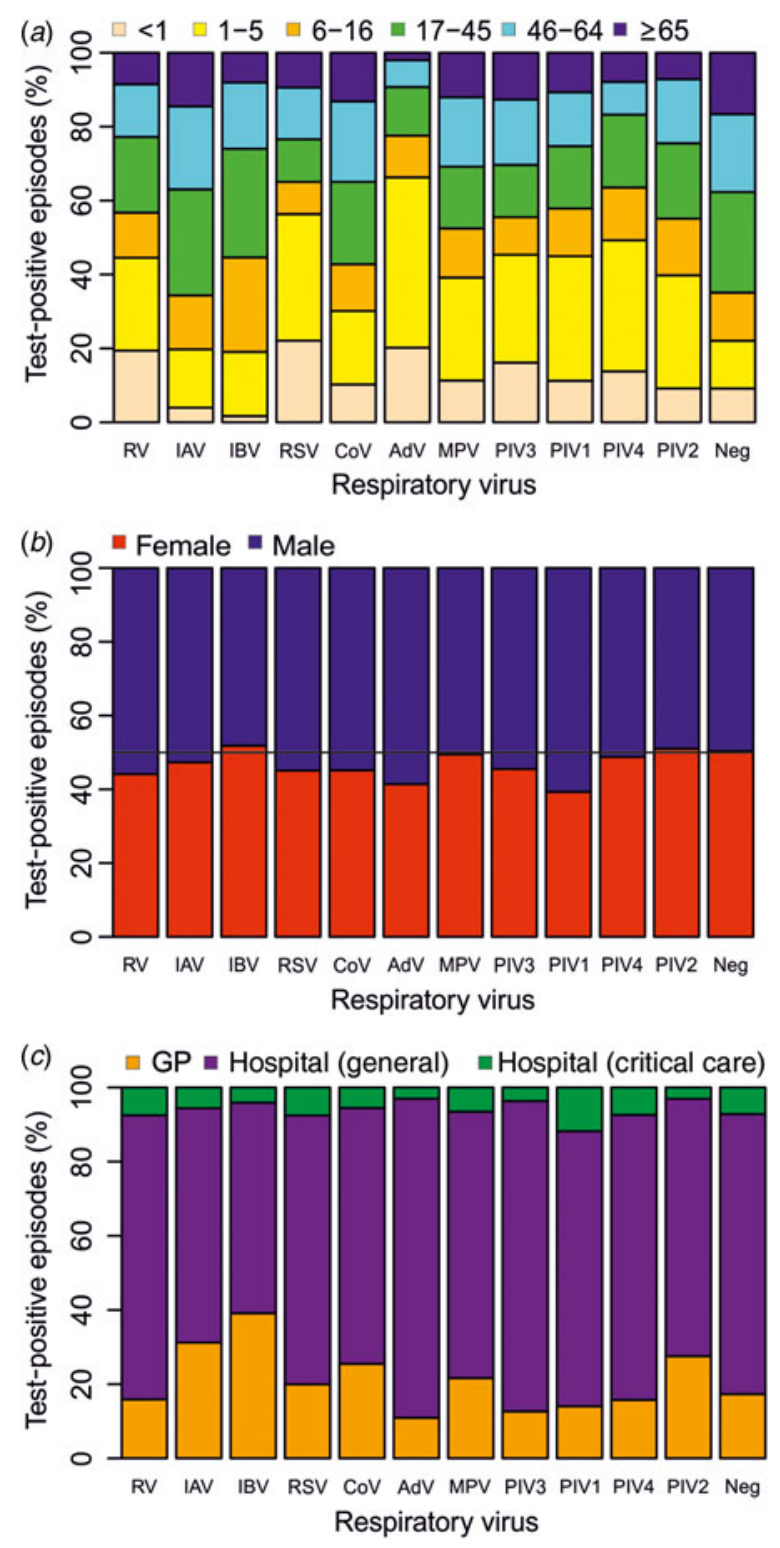

Fig. 2. Episodes of viral respiratory infection by patient subgroup. Distribution of each viral infection and virus-negative illness (Neg) by $(a)$ age group, $(b)$ gender, and $(c)$ patient origin. These results are based on 26974 patient episodes of respiratory illness; excluding patients tested during the major waves of influenza $\mathrm{A}(\mathrm{H} 1 \mathrm{~N} 1)$ pdm09 virus circulation. GP, General Practitioner's surgery; Hospital (general), outpatients and non-critical care patients; Hospital (critical care), patients admitted to an intensive care, intensive therapy, high dependency, or coronary care unit.

attendees (combined proportion: range $31-45 \%$ ), and in January-February in hospital attendees (combined proportion of $30 \%$ ). Of the remaining non-influenza viral infections, a large proportion was attributed to $\mathrm{RSV}, \mathrm{RV}$ and $\mathrm{CoV}$ during periods of high influenza activity; their combined proportions ranged from 39\% to $52 \%$ in GP attendees (December-March) and from $51 \%$ to $55 \%$ in hospital attendees (January-February).

Of 9094 positive patients (in 26974 patients outside of the pandemic period), 1952 were GP attendees, 6560 were general hospital attendees (outpatients and non-critical-care inpatients), and 1282 were inpatients admitted to a critical care ward [an intensive care unit (ICU), intensive therapy unit (ITU), high dependency unit (HDU), or coronary care unit (CCU)]. The latter group provided a proxy for classifying episodes of severe respiratory illness. Eighty-eight percent $(n=4443)$ of GP attendees and 69\% $(n=15027)$ of hospital attendees were aged $>5$ years. As shown in Figure 4, the prevalence of severe episodes in all viruspositive patients, regardless of origin, was greater in patients with RV (7.5\%), RSV (7.5\%), PIV1 (11.8\%) and PIV4 $(7 \cdot 4 \%)$ infections than in virus-negative patients or other viral infections including IAV $(5 \cdot 5 \%)$ and IBV $(4 \cdot 1 \%)$. Investigating further the RV/ IAV and RV/PIV1 comparisons, we found the observed difference in prevalence was statistically significant based on Pearson's $\chi^{2}$ tests $(P=0.036$ and $P=0.05$, respectively). Age-specific prevalence of severe episodes was greatest at the extremes of age $(<5$ and $\geqslant 65$ years) for all viruses except hPIV2 (we note the particularly small sample size for this virus group).

\section{Co-infections and virus mixing patterns}

Of 9654 virus-positive patients from 27284 episodes tested for all 11 viruses, 11\% (1086/9654) had a co-infection. The median age in co-infected patients was 3 years (range $0-91$ years, S.D. $=22$ years) and $58 \%$ were male. Co-infections were more commonly detected in those aged $\leq 5$ years overall $(18 \%$ compared to $7 \%$ in the $>5$ years group) and for each viral infection, particularly RV, RSV, AdV and CoV (detected in $6 \%, 3 \%, 3 \%$ and $2 \%$ of these infections, respectively, in those aged $>5$ years) (Fig. $5 a, b$ ).

A total of 1389 virus pairs were detected in 1086 episodes of co-infection; most episodes involved two viruses $(87 \%, 964 / 1086)$, the remaining involved three $(n=105)$, four $(n=15)$ and five $(n=2)$ viruses. All viruses were detected with most others at least once (Fig. 5c); however, a clustering pattern was evident in which RV, AdV, RSV and CoV were frequently detected with one another. The most common virus detection in a co-infection was RV (56\% of coinfections), the majority of which were with AdV 

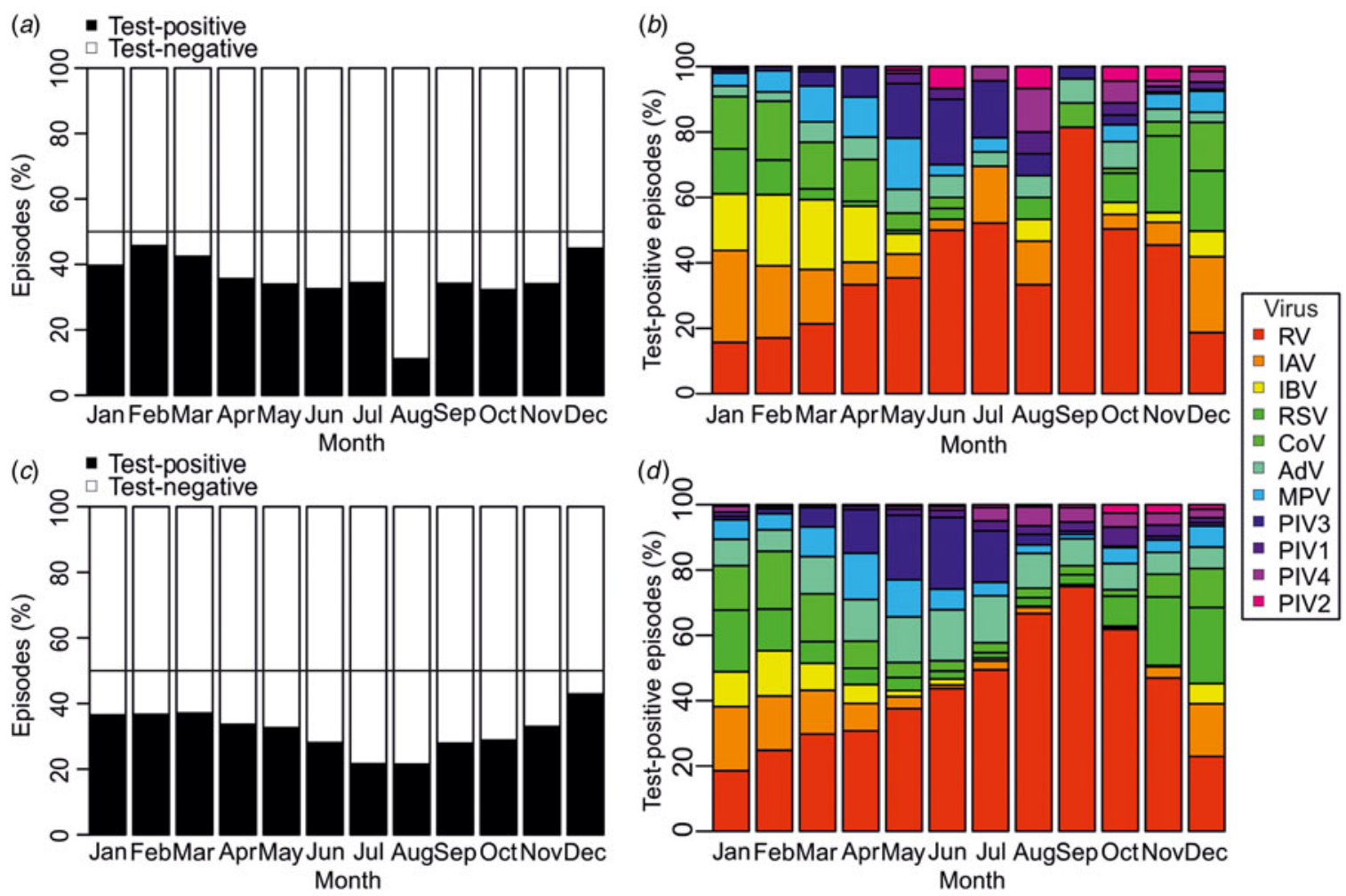

Fig. 3. Distribution of virus-positive/negative episodes of illness and respiratory infection types detected in each calendar month. $(a, b)$ Patients attending primary healthcare services (General Practitioners) and $(c, d)$ patients attending secondary healthcare services (hospital inpatients and outpatients). These results are based on 26974 patient episodes of respiratory illness; excluding patients tested during the major waves of influenza $\mathrm{A}(\mathrm{H} 1 \mathrm{~N} 1) \mathrm{pdm} 09$ virus circulation.

$(n=195,25 \%)$ and RSV $(n=181,23 \%)$. Other viruses relatively frequently detected in co-infections were $\mathrm{AdV}, \mathrm{RSV}$ and $\mathrm{CoV}$; constituting 31\%, 30\% and $28 \%$ of co-infections, respectively.

We found a significant positive correlation between virus detection frequencies in mono-infections and coinfections [Pearson's product-moment correlation $=$ 0.88 (95\% CI $0.60-0.97, P<0.001)$ and fitted linear regression model slope $=0.85 \quad(P<0 \cdot 001)$ ] (Fig. 5d). However, IAV and IBV were identified in co-infections at relatively low frequencies $(n=121$ and $n=68$, respectively) compared to non-influenza viruses (e.g. RV, $n=678$ ) (Fig. 5d).

\section{Factors associated with viral infection and co-infection}

Table 1 summarizes the results of univariable and multivariable logistic regression analyses for associations with viral infection. Season, age group, and patient origin were significantly associated with the odds of viral infection based on unadjusted odds ratio estimates. In the multivariable analysis, several independently significant factors were identified based on the adjusted odds ratios. Viral respiratory infections were more likely to be detected in winter, in children aged 1-5 years, and in GP attendees, irrespective of the other factors. Following adjustment for multiple factors, time period was also a significant predictor (because of a negative confounding by age): the odds of viral infection were significantly greater postpandemic than pre-pandemic.

Significant statistical interactions (based on $P<0.05$ ) revealed that the effect of age was not homogeneous across gender or patient-origin subgroups. This variation in age association across other factors is shown in Figure $6 a, b$ where age-specific infection prevalences are stratified by the third factor. These figures show that the age distribution of infection differed according to gender and patient-origin subgroups.

Table 2 summarizes the results of univariable and multivariable logistic regression analyses for associations with co-infection. Several differences were found in comparison with viral infections overall. Based on unadjusted odds ratio estimates, time period, season (autumn only), age group, gender and patient origin were significantly associated with co-infection. 


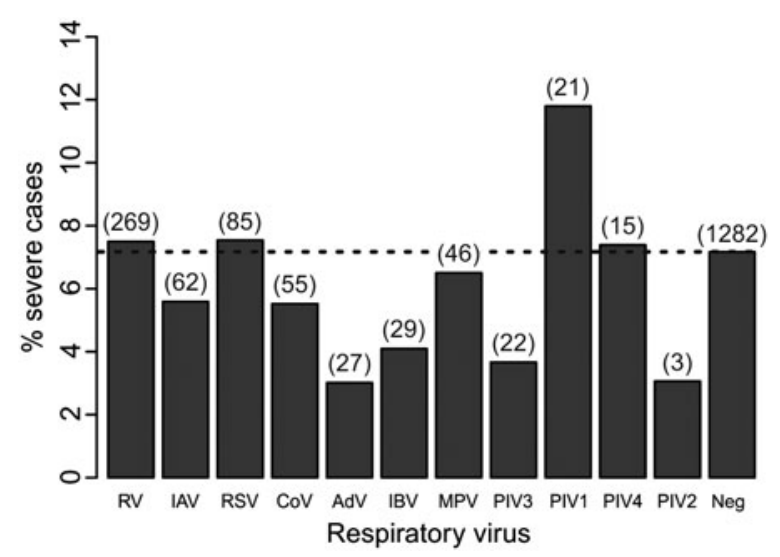

Fig. 4. Prevalence of severe cases in patients with confirmed viral infection attending primary and secondary healthcare facilities in NHS Greater Glasgow and Clyde during 2005-2013. Comparison across viral infection types and virus-negative patients (Neg). Absolute numbers of severe cases are indicated in parentheses. Severe cases were identified based on patients' admission to intensive care, intensive therapy, high dependency or coronary care units.

However, in the multivariable analysis time period and gender were confounded by age and were therefore not identified as significant independent factors. In contrast to viral infection overall, co-infections were equally likely to be detected in spring and winter, were less likely to be detected in the 1-5 years age group than infants, and were more likely to be detected in general hospital attendees (outpatients and those not admitted to critical care wards) than GP attendees.

Significant statistical interactions (based on $P<0.05$ ) revealed that the effect of age on co-infection status was not homogeneous across gender and patient-origin groups. In contrast to viral infection overall, co-infections were relatively more common in males than females in those aged 46-64 years and hospital attendees in all age groups (Fig. $6 c-d$ ).

There was no evidence of a poor model fit based on the global goodness-of-fit tests: (i) $P$ values $=0 \cdot 147$, $0 \cdot 07,0.07$ for the main effect model and two models with interaction terms, respectively, for associations with viral infection overall, and (ii) $P$ values $=0.940$, $0 \cdot 985,0 \cdot 746$ for the main effect model and two models with interaction terms, respectively, for associations with co-infection.

\section{DISCUSSION}

The advent of multiplex PCR as part of routine diagnostics provides an unprecedented opportunity for studying the epidemiology of multiple respiratory viruses simultaneously within a single population. Previous UK-based studies have highlighted the utility of laboratory-based surveillance for monitoring respiratory infection trends, and in comparing the relative burdens between viruses [10,13, 23]. Our study is the first to compare the epidemiologies of different respiratory virus groups utilizing extensive diagnostic data generated by multiplex RT-PCR from patients attending both primary and secondary healthcare services.

The collation of test-negative results by diagnostic laboratories provides valuable denominator information for measuring disease occurrence, to estimate the relative contribution of different pathogens to healthcare usage (such as GP consultations) and to provide an early warning for periods of increased healthcare pressures. Importantly, the diagnostic test data utilized in this study were generated by a single laboratory, permitting a more consistent comparison of trends across patient and virus groups because testing methods were on the whole standardized throughout the study.

Our study has revealed changes in the frequency of virological testing of respiratory illnesses in the NHSGGC health board during 2005-2013, with adults representing an increasingly greater percentage of episodes. However, age-specific prevalences were greater in the 2013 influenza season than in 2005 for all age groups. It is possible that there is raised awareness in the public and/or clinicians, and consequently greater healthcare seeking and/or sampling behaviour in adults. Alternatively these results could reflect a true increase in non-viral causes of respiratory illness in this age group. We note that a shift in the demography of the Glasgow population has been reported [24]. Our observations might indicate the impact of an ageing population on respiratory-related healthcare services, through an increase in GP/hospital consultations, or a genuine increase in the incidence of adult respiratory infections.

RV was the most prevalent virus overall, corroborating previous UK-based studies that include patients attending both primary and secondary healthcare services $[10,12]$. The clinical significance of RV is disputed, although severe cases of disease are recognized depending on virus species, patient subgroups, and season [7, 25-27]. In additional analyses (Fig. 4) we found the prevalence of severe respiratory illness (patients located in critical care wards) was significantly greater in RV infections than IAV, supporting the proposition that $\mathrm{RV}$ is associated with more severe disease than traditionally accepted. 
(a)

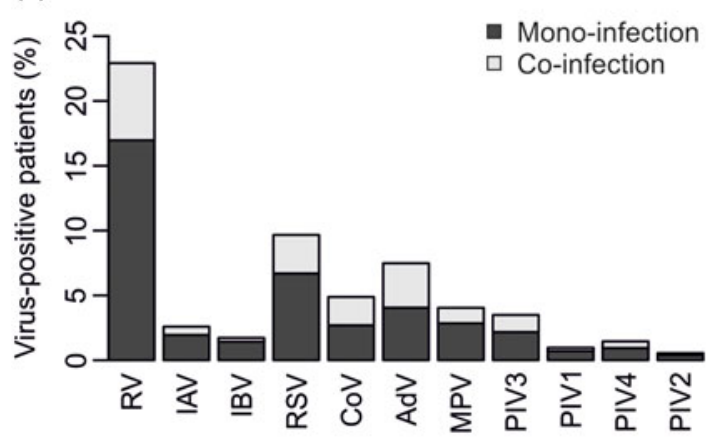

(c)

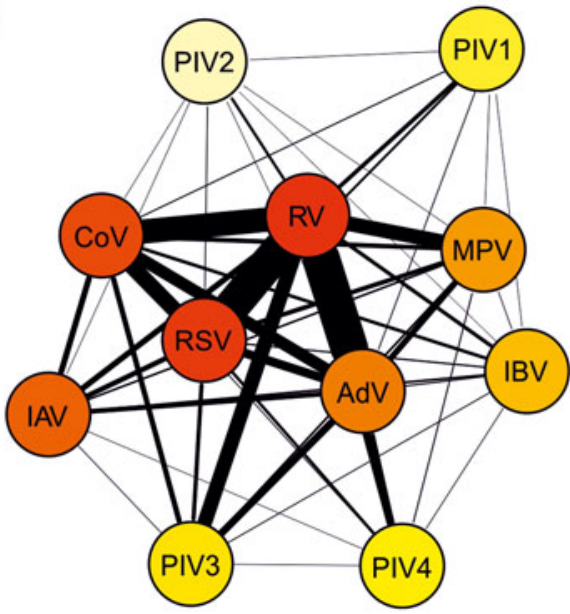

(b)

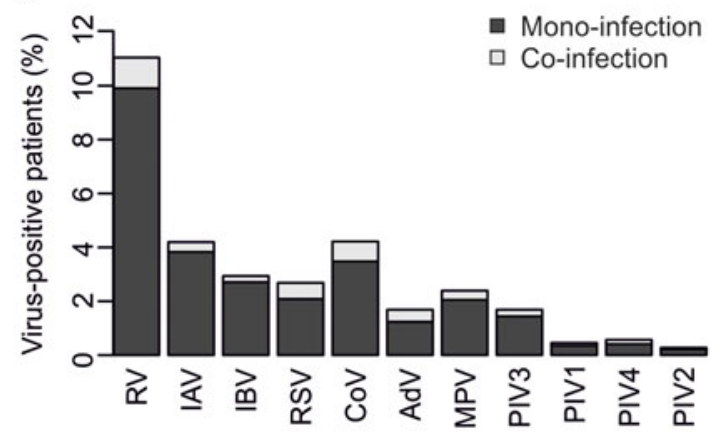

(d)

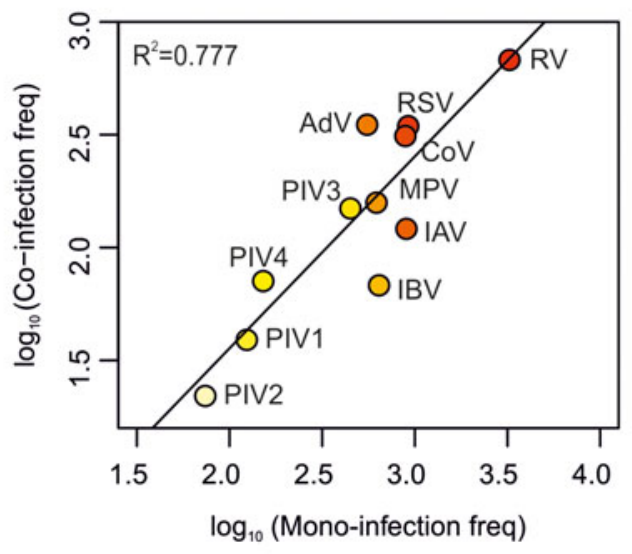

Fig. 5. Co-infection and virus mixing patterns in patients tested for all virus groups. Comparing mono-infection and co-infection distributions for each virus group in $(a)$ children aged $\leqslant 5$ years, and $(b)$ patients aged $>5$ years. $(c)$ A network of co-infections: each node represents a respiratory virus and links between viruses are proportional to the frequency at which each virus pair was observed in co-infected patients. Viruses are coloured according to their prevalence in co-infections (darker represents greater prevalence). $(d)$ Correlation between mono-infection and co-infection frequencies across virus groups; _ _ Fitted linear regression model with corresponding $R^{2}$ value.

Of the other non-influenza viruses, RSV and CoV were relatively highly prevalent. We note that the extent of research into the commonly circulating CoVs is small compared to IAV and RSV, although severe clinical cases are recognized [28]. Our study is the first comparative analysis in the UK to include $\mathrm{CoV}$, providing an important opportunity to quantify its temporal and patient subgroup distributions and co-infection patterns in comparison to the other common virus groups. We confirm that $\mathrm{CoV}$ contributes a large fraction of infections during periods of high influenza activity and that $\mathrm{CoV}$ is relatively frequently co-detected with other viruses. The contribution of different respiratory viruses to the healthcare burden in Scotland has previously been studied [23]. Further investigation on a seasonal basis is needed to help elucidate the public health relevance of $\mathrm{RV}$ and $\mathrm{CoV}$, particularly since $\mathrm{CoV}$ has a similar age distribution as the influenza viruses. The remaining viruses (AdV, MPV, PIV1-4) were detected in comparatively smaller numbers on a yearly basis and during months of high influenza activity.

The 9-year study period provided a novel opportunity to compare the epidemiology of respiratory viruses before and after the 2009 influenza A pandemic [18]. In our multivariable statistical analysis we found viral infections to be more likely in the post-pandemic era. This result was independent of other factors such as patient's age implying non-patient factors, such as a change in the underlying virus population, have increased the likelihood that a patient seeking healthcare services will have a viral infection (as opposed to non-viral causes). Whether this is a direct consequence of the pandemic virus, its impact on the epidemiologies of others viruses, or a consequence of long-term changes in the non-influenza virus population, 
Table 1. Investigating factors associated with viral infection using logistic regression

\begin{tabular}{|c|c|c|c|c|c|c|}
\hline Factor & Level & Summary* & $\begin{array}{l}\text { Virus } \\
\text { positive* }\end{array}$ & $\begin{array}{l}\text { Virus } \\
\text { negative* }\end{array}$ & $\begin{array}{l}\text { Unadjusted OR } \\
(95 \% \text { CI, } P \text { value }) \dagger\end{array}$ & $\begin{array}{l}\text { Adjusted OR } \\
(95 \% \text { CI, } P \text { value })\end{array}$ \\
\hline \multirow[t]{2}{*}{ Time period } & Pre-pandemic & $6296(39)$ & $2090(39)$ & 4206 (39) & Reference & Reference \\
\hline & Post-pandemic & $9961(61)$ & $3315(61)$ & $6646(61)$ & $\begin{array}{c}1.00(0.94-1.07 \\
P=0.912)\end{array}$ & $\begin{array}{c}1 \cdot 31(1.22-1 \cdot 41 \\
P<0.001)\end{array}$ \\
\hline \multirow[t]{4}{*}{ Season } & Winter & $5016(31)$ & $2001(37)$ & $3015(28)$ & Reference & Reference \\
\hline & Spring & $4305(26)$ & $1541(29)$ & $2764(25)$ & $\begin{array}{c}0.84(0.77-0.91 \\
P<0.001)\end{array}$ & $\begin{array}{c}0.79(0.73-0.87 \\
P<0.001)\end{array}$ \\
\hline & Summer & $2952(18)$ & $667(12)$ & $2285(21)$ & $\begin{array}{c}0.44(0.40-0.49 \\
P<0.001)\end{array}$ & $\begin{array}{c}0.42(0.38-0.47 \\
P<0.001)\end{array}$ \\
\hline & Autumn & $3984(25)$ & $1196(22)$ & $2788(26)$ & $\begin{array}{c}0.65(0.59-0.71 \\
P<0.001)\end{array}$ & $\begin{array}{c}0.61(0.56-0.67 \\
P<0.001)\end{array}$ \\
\hline \multirow[t]{6}{*}{ Age group (years) } & $<1$ & 1277 (13) & 959 (18) & $1218(11)$ & Reference & Reference \\
\hline & $1-5$ & $2596(16)$ & $1327(25)$ & $1269(12)$ & $\begin{array}{c}1.33(1 \cdot 18-1 \cdot 49 \\
P<0.001)\end{array}$ & $\begin{array}{c}1 \cdot 25(1 \cdot 11-1 \cdot 41 \\
P<0.001)\end{array}$ \\
\hline & $6-16$ & $1722(11)$ & $564(10)$ & $1158(11)$ & $\begin{array}{c}0.62(0.54-0.71 \\
P<0.001)\end{array}$ & $\begin{array}{c}0.53(0.46-0.60 \\
P<0.001)\end{array}$ \\
\hline & $17-45$ & $3782(23)$ & $1035(19)$ & $2747(25)$ & $\begin{array}{c}0.48(0.43-0.53 \\
P<0.001)\end{array}$ & $\begin{array}{c}0.36(0.32-0.40 \\
P<0.001)\end{array}$ \\
\hline & $46-64$ & $3247(20)$ & $866(16)$ & $2381(22)$ & $\begin{array}{c}0.46(0 \cdot 41-0.52 \\
P<0 \cdot 001)\end{array}$ & $\begin{array}{c}0 \cdot 37(0 \cdot 33-0 \cdot 41 \\
P<0 \cdot 001)\end{array}$ \\
\hline & $\geqslant 65$ & $2733(17)$ & $654(12)$ & 2079 (19) & $\begin{array}{c}0.40(0 \cdot 35-0.45 \\
P<0 \cdot 001)\end{array}$ & $\begin{array}{c}0.34(0 \cdot 30-0 \cdot 39 \\
P<0.001)\end{array}$ \\
\hline \multirow[t]{2}{*}{ Gender } & Female & 7941 (49) & $2575(48)$ & $5366(49)$ & Reference & Reference \\
\hline & Male & $8316(51)$ & $5486(52)$ & $2830(51)$ & $\begin{array}{c}1 \cdot 07(1 \cdot 01-1 \cdot 15 \\
P=0 \cdot 03)\end{array}$ & $\begin{array}{c}1 \cdot 08(1 \cdot 01-1 \cdot 15 \\
P=0.032)\end{array}$ \\
\hline \multirow[t]{3}{*}{ Patients' origin $\S$} & GP & 3012 (19) & $1260(23)$ & $1752(16)$ & Reference & Reference \\
\hline & $\begin{array}{l}\text { Hospital } \\
\text { (general) }\end{array}$ & $11878(73)$ & $3725(69)$ & $8153(75)$ & $\begin{array}{c}0.64(0.59-0.69 \\
P<0.001)\end{array}$ & $\begin{array}{c}0.54(0.49-0.59 \\
P<0.001)\end{array}$ \\
\hline & $\begin{array}{l}\text { Hospital } \\
\text { (critical care) }\end{array}$ & $1367(8)$ & $420(8)$ & $947(9)$ & $\begin{array}{c}0.62(0.54-0.71 \\
P<0.001)\end{array}$ & $\begin{array}{c}0.56(0.49-0.65 \\
P<0.001)\end{array}$ \\
\hline
\end{tabular}

OR, Odds ratio; CI, confidence interval.

* Distribution of patient numbers, with corresponding $\%$ in parentheses, across factor levels for all patients (summary) and for virus-positive and virus-negative groups.

$\dagger$ Unadjusted OR based on univariable logistic regression.

$\$$ Adjusted OR based on multivariable logistic regression.

§ Patients' location corresponding with first clinical sample: GP, General Practitioner's surgery; Hospital (general), outpatients and non-critical-care patients; Hospital (critical care), patients admitted to an intensive care, intensive therapy, high dependency, or coronary care unit.

remains to be elucidated. Seasonal and patient-related factors corroborate existing knowledge and were independent of time, indicating the generality of these factors as predictors of viral infection.

It is well recognized that the burden of viral respiratory illness lies predominantly in young children [29]. We found that in patients with respiratory illness attending healthcare facilities, those aged 1-5 years were more likely than other age groups to have a viral infection independent of season or time period. The most commonly detected viruses in this age group were RV, RSV, AdV and MPV (20\%, 9.3\%, $9 \cdot 1 \%$ and $4 \cdot 7 \%$ of infections, respectively) corroborating previous reports $[23,30]$. Together with a recent study that found bacterial-viral co-infections were relatively uncommon in children with pneumonia [31], these findings support the concern regarding the overprescription of antibiotics in children [32]. That the increasing trend in virus prevalence was most notable in infants $(<1$ year) also warrants further attention. While it is possible that these findings are influenced by changes in clinical testing decisions, we note that this trend is particularly pertinent in relation to recent European outbreaks of enterovirus D68 in children [33]; investigation into the contribution of individual viruses will be the focus of future work. We further 
(a)
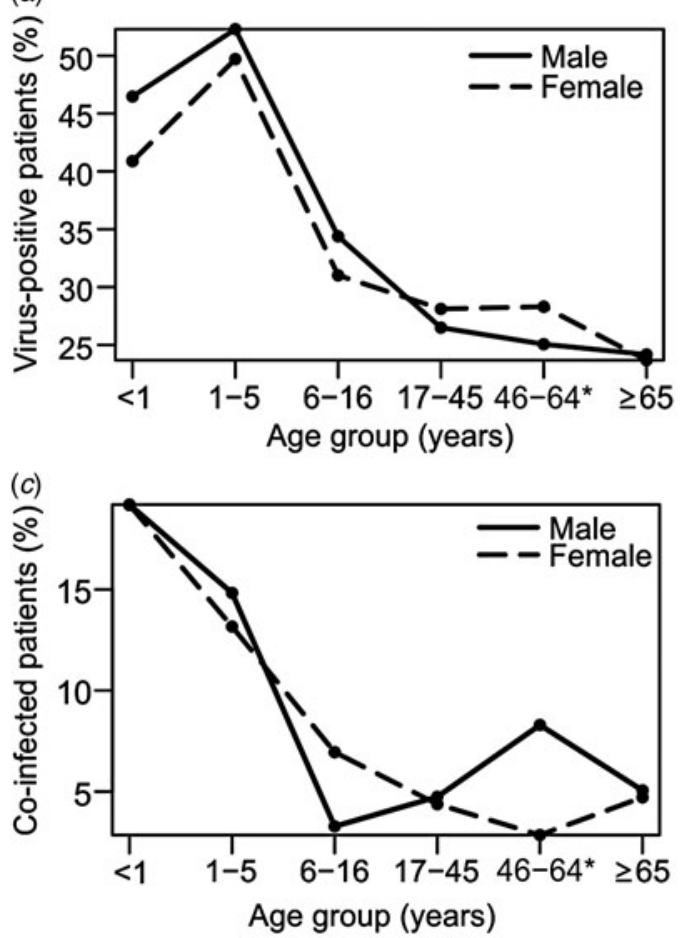

(b)
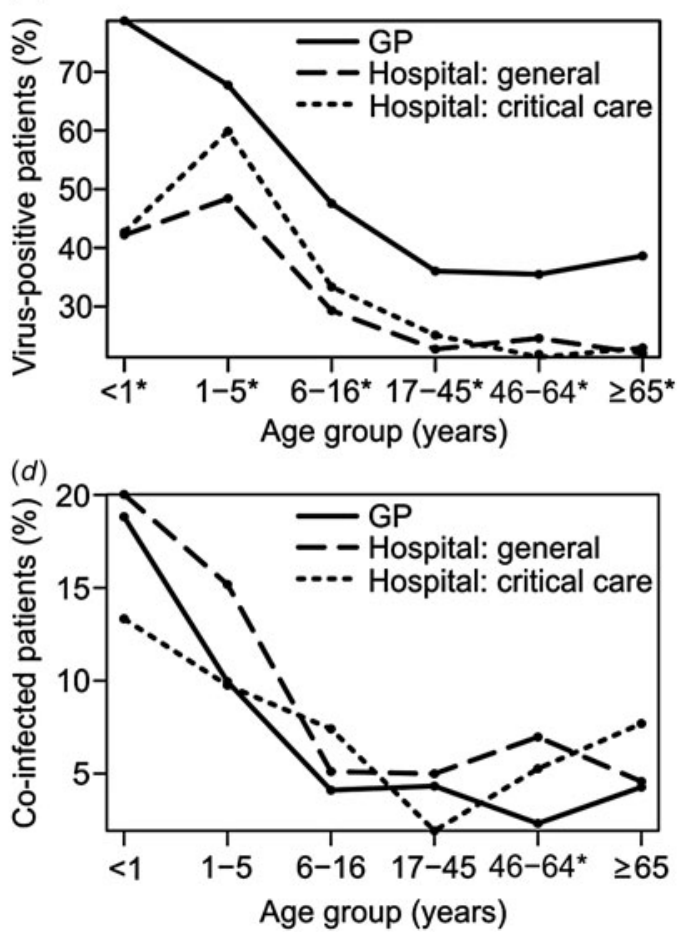

Fig. 6. Stratification of viral infection and co-infection associations. Age-specific viral infection $(a, b)$ and co-infection $(c, d)$ prevalences stratified by gender and patient origin. Significant interactions with age are indicated by an asterisk $(*)$.

note that, based on the multivariable statistical analyses, the increasing trend in prevalence in children explained why co-infections were more likely detected in the post-2009 pandemic era.

There are very few studies describing co-infection patterns in respiratory viruses. Our study provides the largest examination to date, confirming that around $11 \%$ of viral infections in patients attending healthcare services in an urban setting involve more than one virus, similar to the $10 \cdot 4 \%$ reported by a previous UK-based study [12]. That nearly all respiratory viruses were co-detected with all other viruses highlights the sufficient opportunities for co-infections. We would expect co-infection frequencies to reflect individual virus prevalences. Indeed, in line with the aforementioned study [12], RV was the most common detection in co-infections, RV/RSV was a frequent pairing, and most co-infections were in children aged $<5$ years. Our study also reveals that CoVs are relatively frequently involved in co-infections. However, co-infections with influenza viruses were relatively few, perhaps explained by differences in their age and seasonal distributions, or an inter-viral interference [34].
We found that the average age of co-infection was 3 years, compared to 17 years for viral infections overall, and co-infections were more likely in infants than in those aged 1-5 years. That co-infections were more likely in young children is probably explained by (i) a greater opportunity for co-infection due to a shorter exposure lifetime and consequently greater susceptibility to a wider array of viruses, and (ii) a greater chance of co-infections being detected because children tend to shed virus for longer periods.

In adults, the age distribution of co-infections differed according to gender and patient origin; the prevalence was greatest in males and in general hospital attendees not admitted to critical care wards for those aged 46-64 years (Fig. $6 c, d$ ). This result provides insight into an age-dependent factor in co-infection patterns in adults but must be viewed with some caution; it is potentially influenced by a bias in multiple specimens submitted in relation to single episodes of illness in adults, most likely as a result of comorbidities. Interestingly, co-infections were more likely in general hospital attendees not admitted to critical care wards than GP attendees, supporting the potential role of co-infections in illness severity [35]. 
Table 2. Investigating factors associated with co-infection using logistic regression

\begin{tabular}{|c|c|c|c|c|c|c|}
\hline Factor & Level & Summary* & Co-infection* & $\begin{array}{l}\text { Mono- } \\
\text { infection* }\end{array}$ & $\begin{array}{l}\text { Unadjusted OR } \\
(95 \% \mathrm{CI}, P \text { value }) \dagger\end{array}$ & $\begin{array}{l}\text { Adjusted OR } \\
(95 \% \mathrm{CI}, P \text { value })\end{array}$ \\
\hline \multirow[t]{2}{*}{ Time period } & Pre-pandemic & $2090(39)$ & $232(44)$ & $1858(38)$ & Reference & Reference \\
\hline & Post-pandemic & $3315(61)$ & $293(56)$ & $3022(62)$ & $\begin{array}{c}0.78(0.65-0.93 \\
P=0.006)\end{array}$ & $\begin{array}{c}0.97(0 \cdot 80-1 \cdot 18 \\
P=0 \cdot 774)\end{array}$ \\
\hline \multirow[t]{4}{*}{ Season } & Winter & $2001(37)$ & $209(40)$ & $1792(37)$ & Reference & Reference \\
\hline & Spring & $1541(29)$ & $165(31)$ & $1376(28)$ & $\begin{array}{c}1 \cdot 03(0 \cdot 83-1 \cdot 28 \\
P=0 \cdot 801)\end{array}$ & $\begin{array}{c}0 \cdot 94(0 \cdot 75-1 \cdot 18 \\
P=0 \cdot 595)\end{array}$ \\
\hline & Summer & $667(12)$ & $54(10)$ & $613(13)$ & $\begin{array}{c}0.76(0.55-1.03 \\
P=0.079)\end{array}$ & $\begin{array}{c}0.55(0 \cdot 40-0 \cdot 76 \\
P<0 \cdot 001)\end{array}$ \\
\hline & Autumn & $1196(22)$ & $97(18)$ & $1099(23)$ & $\begin{array}{c}0.76(0.59-0.97 \\
P=0.03)\end{array}$ & $\begin{array}{c}0 \cdot 63(0 \cdot 48-0 \cdot 82 \\
P=0 \cdot 001)\end{array}$ \\
\hline \multirow[t]{6}{*}{ Age group (years) } & $<1$ & 959 (18) & $184(35)$ & 775 (16) & Reference & Reference \\
\hline & $1-5$ & $1327(25)$ & $187(36)$ & $1140(23)$ & $\begin{array}{c}0 \cdot 69(0 \cdot 55-0 \cdot 86 \\
P=0 \cdot 001)\end{array}$ & $\begin{array}{c}0.67(0.54-0 \cdot 84 \\
P=0 \cdot 001)\end{array}$ \\
\hline & $6-16$ & $546(10)$ & $28(5)$ & $536(11)$ & $\begin{array}{c}0 \cdot 22(0 \cdot 15-0 \cdot 33 \\
P<0 \cdot 001)\end{array}$ & $\begin{array}{c}0 \cdot 21(0 \cdot 14-0 \cdot 32 \\
P<0 \cdot 001)\end{array}$ \\
\hline & $17-45$ & 1035 (19) & $47(9)$ & $988(20)$ & $\begin{array}{c}0 \cdot 20(0 \cdot 14-0 \cdot 28 \\
P<0 \cdot 001)\end{array}$ & $\begin{array}{c}0 \cdot 21(0 \cdot 15-0 \cdot 30 \\
P<0 \cdot 001)\end{array}$ \\
\hline & $46-64$ & $866(16)$ & $47(9)$ & 819 (17) & $\begin{array}{c}0 \cdot 24(0 \cdot 17-0 \cdot 34 \\
P<0 \cdot 001)\end{array}$ & $\begin{array}{c}0 \cdot 24(0 \cdot 17-0 \cdot 34 \\
P<0 \cdot 001)\end{array}$ \\
\hline & $\geqslant 65$ & $654(12)$ & $32(6)$ & $622(13)$ & $\begin{array}{c}0 \cdot 22(0 \cdot 15-0 \cdot 32 \\
P<0 \cdot 001)\end{array}$ & $\begin{array}{c}0 \cdot 21(0 \cdot 14-0 \cdot 31 \\
P<0 \cdot 001)\end{array}$ \\
\hline \multirow[t]{2}{*}{ Gender } & Female & $2575(48)$ & $222(42)$ & $2353(48)$ & Reference & Reference \\
\hline & Male & $2830(52)$ & $303(58)$ & $2527(52)$ & $\begin{array}{c}1 \cdot 27(1 \cdot 06-1 \cdot 52 \\
P=0 \cdot 01)\end{array}$ & $\begin{array}{c}1 \cdot 11(0 \cdot 92-1 \cdot 33 \\
P=0 \cdot 293)\end{array}$ \\
\hline \multirow[t]{3}{*}{ Patients' origin $\S$} & GP & $1260(23)$ & 72 (14) & $1188(24)$ & Reference & Reference \\
\hline & $\begin{array}{l}\text { Hospital } \\
\text { (general) }\end{array}$ & $3725(69)$ & $418(80)$ & $3307(68)$ & $\begin{array}{c}2 \cdot 09(1 \cdot 61-2 \cdot 70 \\
P<0 \cdot 001)\end{array}$ & $\begin{array}{c}1 \cdot 52(1 \cdot 15-2 \cdot 00 \\
P=0 \cdot 003)\end{array}$ \\
\hline & $\begin{array}{l}\text { Hospital } \\
\text { (critical care) }\end{array}$ & $420(8)$ & $35(7)$ & $385(8)$ & $\begin{array}{c}1 \cdot 50(0 \cdot 99-2 \cdot 28 \\
P=0 \cdot 058)\end{array}$ & $\begin{array}{l}1 \cdot 15(0 \cdot 75-1 \cdot 79 \\
P=0 \cdot 521)\end{array}$ \\
\hline
\end{tabular}

OR, Odds ratio; $\mathrm{CI}$, confidence interval.

* Distribution of patient numbers, with corresponding $\%$ in parentheses, across factor levels for all patients (summary) and for co-infection and mono-infection groups.

$\dagger$ Unadjusted OR based on univariable logistic regression.

$\$$ Adjusted OR based on multivariable logistic regression.

§ Patients' location corresponding with first clinical sample: GP, General Practitioner's surgery; Hospital (general), outpatients and non-critical-care patients; Hospital (critical care), patients admitted to an intensive care, intensive therapy, high dependency, or coronary care unit.

There are several limitations to our study to be noted. Detection of viral nucleic acid may not represent active infection for all viruses in all cases [36], potentially introducing detection biases temporally and across patient groups. Furthermore, the timing of infection events, and variation in shedding duration across virus and patient groups [37, 38], could potentially bias the observed co-infection patterns. We also note that our study lacked information on the presence/absence of bacterial pathogens which are also significant contributors to respiratory infections.

One further important consideration is that laboratory diagnostic data cannot inform on the epidemiology of asymptomatic infections in the community, or in symptomatic people who do not attend healthcare services. Furthermore, that viral populations are not static could also impact on the generalisability of the observed trends and associations; the introduction of new strains can alter disease outcomes, and consequently healthcare seeking behaviour, influencing the stability of healthcare consultation rates in patient subgroups. Given the dynamic nature of virus populations, the epidemiological information generated through surveillance must be maintained to ensure future vaccine and antiviral developments are directed to where they are most needed $[39,40]$. 


\section{CONCLUSIONS}

Our study provides the most comprehensive description of viral respiratory infections in the UK to date, revealing new epidemiological insights with public health relevance. Of particular concern is a greater viral prevalence in 2013 compared to 2005, particularly in infants, and a greater risk of viral infection in the post-2009 pandemic era. Further investigation into the long-term temporal dynamics of individual viruses and the epidemiological consequences of virus cocirculation is needed.

\section{SUPPLEMENTARY MATERIAL}

For supplementary material accompanying this paper visit http://dx.doi.org/10.1017/S0950268816000339.

\section{ACKNOWLEDGEMENTS}

The authors are grateful to Dominic Mellor, Emma Thomson, Louise Matthews and Richard Reeve for their helpful critique of the manuscript and discussions. A subset of the clinical samples was provided by Health Protection Scotland as part of the Scottish Enhanced Respiratory Viral Infection Surveillance programme.

This work was supported by the Medical Research Council UK (Grant G0801822).

\section{DECLARATION OF INTEREST}

None.

\section{REFERENCES}

1. Nair H, et al. Global burden of respiratory infections due to seasonal influenza in young children: a systematic review and meta-analysis. Lancet 2011; 378: 1917-1930.

2. Nair H, et al. Global burden of acute lower respiratory infections due to respiratory syncytial virus in young children: a systematic review and meta-analysis. Lancet 2010; 375: 1545-1555.

3. Murray CJ, et al. Disability-adjusted life years (DALYs) for 291 diseases and injuries in 21 regions, 1990-2010: a systematic analysis for the Global Burden of Disease Study 2010. Lancet 2012; 380: 2197-2223.

4. Makela MJ, et al. Viruses and bacteria in the etiology of the common cold. Journal of Clinical Microbiology 1998; 36: 539-542.

5. Clark TW, et al. Adults hospitalised with acute respiratory illness rarely have detectable bacteria in the absence of COPD or pneumonia; viral infection predominates in a large prospective UK sample. Journal of Infection 2014; 69: 507-515.

6. Head MG, et al. Investments in respiratory infectious disease research 1997-2010: a systematic analysis of UK funding. British Medical Journal Open 2014; 4: e004600

7. Bizzintino J, et al. Association between human rhinovirus $\mathrm{C}$ and severity of acute asthma in children. European Respiratory Journal 2011; 37: 1037-1042.

8. Panda S, et al. Human metapneumovirus: review of an important respiratory pathogen. International Journal of Infectious Diseases 2014; 25: 45-52.

9. Kuiken T, et al. Newly discovered coronavirus as the primary cause of severe acute respiratory syndrome. Lancet 2003; 362: 263-270.

10. Zhao H, et al. A new laboratory-based surveillance system (Respiratory DataMart System) for influenza and other respiratory viruses in England: results and experience from 2009 to 2012. Eurosurveillance 2014; 19: 1-10.

11. Brittain-Long $\mathbf{R}$, et al. Seasonal variations of 15 respiratory agents illustrated by the application of a multiplex polymerase chain reaction assay. Scandinavian Journal of Infectious Diseases 2012; 44: 9-17.

12. Goka EA, et al. Single, dual and multiple respiratory virus infections and risk of hospitalization and mortality. Epidemiology and Infection 2015; 143: 37-47.

13. Pitman RJ, et al. Assessing the burden of influenza and other respiratory infections in England and Wales. Journal of Infection 2007; 54: 530-538.

14. Zhang D, et al. Epidemiology characteristics of respiratory viruses found in children and adults with respiratory tract infections in southern China. International Journal of Infectious Diseases 2014; 25: 159-164.

15. Feng L, et al. Viral etiologies of hospitalized acute lower respiratory infection patients in China, 2009-2013. PLoS ONE 2014; 9: e99419.

16. Minodier L, et al. Epidemiology and viral etiology of the influenza-like illness in corsica during the 2012 2013 Winter: an analysis of several sentinel surveillance systems. PLoS ONE 2014; 9: e100388.

17. Information Services Division (ISD) Scotland. Population estimates (http://www.isdscotland.org/Products-andServices/GPD-Support/Population/Estimates/). Accessed 2 November 2015.

18. Yang L, et al. Impact of the $2009 \mathrm{H} 1 \mathrm{~N} 1$ pandemic on age-specific epidemic curves of other respiratory viruses: a comparison of pre-pandemic, pandemic and postpandemic periods in a subtropical city. PLOS ONE 2015; 10: e0125447.

19. NHS Greater Glasgow and Clyde. West of Scotland Specialist Virology Centre (http://www.nhsggc.org.uk/ content/default.asp?page=home_virology). Accessed 2 November 2015.

20. Gunson RN, Carman WF. During the summer 2009 outbreak of 'swine flu' in Scotland what respiratory pathogens were diagnosed as $\mathrm{H} 1 \mathrm{~N} 1 / 2009$ ? BMC Infectious Diseases 2011; 11: 192.

21. le Cessie S, van Houwelingen J. A goodness-of-fit test for binary regression models, based on smoothing methods. Biometrics 1991; 47: 1267-1282. 
22. R Core Team. R: A language and environment for statistical computing. R Foundation for Statistical Computing, Vienna, Austria (http://www.R-project.org/). Accessed 2 November 2015.

23. Gaunt ER, et al. Disease burden of the most commonly detected respiratory viruses in hospitalized patients calculated using the disability adjusted life year (DALY) model. Journal of Clinical Virology 2011; 52: 215-221.

24. Glasgow Centre for Population Health. Population trends by age group in Glasgow, 1981-2013 (http:// www.understandingglasgow.com/indicators/population/ trends/trends_by_age_group). Accessed 2 November 2015.

25. Lee WM, et al. Human rhinovirus species and season of infection determine illness severity. American Journal of Respiratory and Critical Care Medicine 2012; 186: 886891.

26. Lau SK, et al. Clinical features and complete genome characterization of a distinct human rhinovirus (HRV) genetic cluster, probably representing a previously undetected HRV species, HRV-C, associated with acute respiratory illness in children. Journal of Clinical Microbiology 2007; 45: 3655-3664.

27. Rahamat-Langendoen $\mathbf{J C}$, et al. The significance of rhinovirus detection in hospitalized children: clinical, epidemiological and virological features. Clinical Microbiology and Infection 2013; 19: E435-442.

28. Gaunt ER, et al. Epidemiology and clinical presentations of the four human coronaviruses 229E, HKU1, NL63, and OC43 detected over 3 years using a novel multiplex real-time PCR method. Journal of Clinical Microbiology 2010; 48: 2940-2947.

29. Tregoning JS, Schwarze J. Respiratory viral infections in infants: causes, clinical symptoms, virology, and immunology. Clinical Microbiology Reviews 2010; 23: 74-98.

30. Tanner H, Boxall E, Osman H. Respiratory viral infections during the 2009-2010 winter season in Central
England, UK: incidence and patterns of multiple virus co-infections. European Journal of Clinical Microbiology and Infectious Diseases 2012; 31: 3001-3006.

31. Jain $\mathbf{S}$, et al. Community-acquired pneumonia requiring hospitalization among U.S. children. New England Journal of Medicine 2015; 372: 835-845.

32. Kool M, et al. Respiratory virus infections in febrile children presenting to a general practice out-of-hours service. European Journal of General Practice 2015; 21: 5-11.

33. Meijer A, et al. Emergence and epidemic occurrence of enterovirus 68 respiratory infections in The Netherlands in 2010. Virology 2012; 423: 49-57.

34. Greer RM, et al. Do rhinoviruses reduce the probability of viral co-detection during acute respiratory tract infections? Journal of Clinical Virology 2009; 45: 10-15.

35. Goka EA, et al. Single and multiple respiratory virus infections and severity of respiratory disease: a systematic review. Paediatric Respiratory Reviews 2014; 15: 363 370.

36. Rhedin S, et al. Clinical utility of PCR for common viruses in acute respiratory illness. Pediatrics 2014; 133: e538-545.

37. Munywoki PK, et al. Influence of age, severity of infection, and co-infection on the duration of respiratory syncytial virus (RSV) shedding. Epidemiology and Infection 2015; 143: 804-812.

38. Martin ET, et al. Epidemiology of multiple respiratory viruses in childcare attendees. Journal of Infectious Diseases 2013; 207: 982-989.

39. Gillim-Ross L, Subbarao K. Emerging respiratory viruses: challenges and vaccine strategies. Clinical Microbiology Reviews 2006; 19: 614-636.

40. Nichols WG, Peck Campbell AJ, Boeckh M. Respiratory viruses other than influenza virus: impact and therapeutic advances. Clinical Microbiology Reviews 2008; 21: 274-290. 